Psychotherapeut 2014 $\cdot 59: 437-438$

DOI 10.1007/s00278-014-1089-6

Online publiziert: 23. November 2014

(c) Springer-Verlag Berlin Heidelberg 2014
Harald J. Freyberger ${ }^{1} \cdot$ Wolfgang Schneider ${ }^{2}$

${ }^{1}$ Klinik und Poliklinik für Psychiatrie und Psychotherapie, Universitätsmedizin Greifswald am Helios-Hanseklinikum Stralsund

${ }^{2}$ Klinik und Poliklinik für Psychosomatik und Psychotherapeutische Medizin, Universität Rostock

\title{
Relevanz der Diagnostik in der Psychotherapie
}

Mit der Veröffentlichung der 5. Auflage des Diagnostic and Statistical Manual of Mental Disorders (DSM-5) ist die Diskussion um diagnostische Standards, Methoden und Ziele wieder entfacht worden, nachdem in den frühen 1990er Jahren mit der Einführung des DSM-IV und der 10. Ausgabe der International Statistical Classification of Diseases and Related Health Problems (ICD-10) bereits eine breite Debatte geführt worden war (Schneider u. Freyberger 1994). Die damalige Diskussion wurde durch die Operationalisierung diagnostischer Kategorien geprägt und die damit verbundene Verankerung der Diagnosen in vergleichsweise einfach beobachtbaren Symptomen unter Vernachlässigung komplexerer diagnostischer Merkmale. Als ein Ergebnis der damals stattfindenden Debatte kann die zunehmende Operationalisierung weiterer, in der Psychotherapie u. a. für die Indikationsstellung, die Fokusbild und die Evaluation relevanter Merkmale betrachtet werden, wie sie z. B. mit der Operationalisierten Psychodynamischen Diagnostik (OPD; Arbeitskreis OPD 1996) vorgelegt wurde.

Die derzeitigen kritischen Beiträge zum DSM-5 weisen insbesondere auf die Pathologisierungstendenzen der operationalisierten psychiatrischen Diagnosenmodelle über die Schaffung immer neuer Erkrankungen und die Herabsenkung der diagnostischen Schwellen hin, die für eine Diagnose als konstituierend angesehen werden (Stieglitz u. Freyberger 2013; Stieglitz u. Hiller 2013). Andere Autoren greifen eher einzelne diagnostische Kategorien des DSM-5 sowie deren Definition und Operationalisierung auf, wie es Hiller u. Rief in dieser Ausgabe der Zeitschrift Psychotherapeut für das neu eingeführte Konzept der ,somatic symptom disorder" vornehmen und die Abschaffung der somatoformen Störung im DSM-5 kritisieren.

Mit einem im engeren Sinn konkreten diagnostischen Thema beschäftigt sich im vorliegenden Heft eine Arbeitsgruppe um Plener aus der kinder- und jugendpsychiatrischen Klinik der Universität Ulm, Domhardt et al., die im Rahmen einer Pilotstudie ein neues Screeninginstrument zu Autismusspektrumstörungen vorstellt, die im DSM- 5 bezüglich der Kriterien ebenfalls revidiert wurden.

Und die Entwicklung der psychiatrischen Diagnostik weist eine Vielzahl an kritischen Aspekten auf, die unter der Ära des diagnostischen und therapeutischen Pragmatismus sowie der Orientierung an Standards der „evidence based medicine"/Psychotherapie viel zu wenig reflektiert werden. Dazu gehört einmal die Fokussierung von Symptomen und Syndromen, bei der diesen zugrunde liegende psychosoziale Bedingungen weitgehend ausgeblendet werden, eine programmatische biologistische Orientierung, die zu einem „Maschinenbild“ des Menschen führt sowie die individuelle biografische Gewordenheit und Sinnhaftigkeit von "gestörtem“ Erleben und Verhalten ausgrenzt. Die bloße Exploration von Symptomen und Symptomkonfigurationen führt dazu, dass die Beziehung und Beziehungsherstellung im diagnostischen Prozess vernachlässigt werden, was natürlich auch Auswirkungen auf die psychotherapeutische Arbeit hat. Schneider u. Freyberger diskutieren entsprechende Aspekte in ihrem Aufsatz in diesem Heft.

Die Favorisierung der operationalisierten Diagnosensysteme und ihre Konsequenzen für die Psychotherapie greift der ehemalige Chairman des DSM-IV Allen Frances in einem engagierten Statement auf, bei dem er die Bevorzugung verhaltenstherapeutischer Methoden, aber auch psychopharmakologischer Ansätze in der Therapie von "leichteren" psychischen Erkrankungen herausarbeitet und kritisiert.

Es bleibt für die wissenschaftliche und praktische Psychotherapie zu wünschen, dass zukünftig wieder ein Mehr an kritischer Reflexion über das „Menschenbild“, die Konzeptualisierung von psychischen und psychosomatischen Erkrankungen und den Beziehungsaspekt in der Diagnostik und Psychotherapie vollzogen wird, da ansonsten die Gefahr besteht, dass Psychotherapie zu einem schlichten Reparaturbetrieb für abweichendes Verhalten wird. In den letzten 20 Jahren hat sich diese Tendenz deutlich abgezeichnet. Neben den administrativen und versorgungspolitischen Rahmenbedingungen hat sich über die Implementierung von Ausbildungsgängen zum psychologischen Psychotherapeuten über deren hohes Ausmaß an Strukturierung und Formalisierung der Curricula auch eine andere Generation an Psychotherapeuten herausgebildet, die Gefahr läuft, Psychotherapie als eine insbesondere über den Erwerb von Wissen und psychotherapeutischen Techniken orientierte Profession zu verstehen. Die „modernen“ psychiatrischen Diagnosensysteme stellen dafür ein typisches Rüstzeug dar, aus dem sich dann für die entsprechenden "Störungs- 
bilder" möglichst manualisierte spezifische Interventionen erlernen und anwenden lassen. Dieses pragmatische Grundverständnis von Psychotherapie kann zum Verlust der Sensibilität für die emotionale und beziehungsorientierte Dynamik von Psychotherapie führen. Diagnostik stellt keine Einpunkterhebung dar, sondern bleibt Bestandteil des therapeutischen Prozesses, soweit sich das diagnostische Verständnis nicht nur auf Symptome ausrichtet, sondern auch diesen zugrunde liegende psychosoziale Aspekte berücksichtigt. Und diese stellen sich vielfach in der Beziehungsgestaltung und szenischen Gegebenheiten zwischen dem Patienten und dem Psychotherapeuten dar. Um diese Dimension aktiv zu gestalten, muss der Therapeut ein hohes Ausmaß an beziehungsorientierten Kompetenzen und Empathie, für die Empfindungen, Wünsche, Ängste oder auch die Abwehr des Patienten aufweisen. Dies bedeutet, dass der Therapeut Respekt und Akzeptanz für das Subjekt und die individuelle Sicht des Patienten aufweisen sollte. Eine Haltung, die zum einen in der Persönlichkeit des Therapeuten über seine biografische Entwicklung verankert sein wird, die sich aber auch über die professionelle Sozialisation - deren Ausbildungsinhalte sowie den Erfahrungen mit ihren Ausbildern und deren „Tun“ - herleitet. Und hier steht zu befürchten, dass die psychiatrischen Klassifikationsmodelle, aber auch eine Übermanualisierung der therapeutischen Interventionen die benannten subjekt- und beziehungsorientierten Dimensionen vernachlässigt. Und wir über deren „bloße“ Anwendung die Sensibilität und Aufmerksamkeit für diese verlernen, soweit wir diese gehabt haben. Unsere Ausbildungskandidaten würden dann diese „essentials“ von Diagnostik und Therapie in unserem Feld nicht oder nur wenig kennenlernen.

\section{Korrespondenzadresse}

Prof. Dr. Harald J. Freyberger

Klinik und Poliklinik für Psychiatrie und Psychotherapie, Universitätsmedizin Greifswald am Helios-Hanseklinikum Stralsund Rostocker Chaussee 70, 18437 Stralsund freyberg@uni-greifswald.de

\section{Literatur}

Arbeitskreis OPD (Hrsg 1996) Operationalisierte Psychodynamische Diagnostik. Grundlagen und Manual, 1. korr. Auflage. Huber, Bern

Schneider W, Freyberger HJ (1994) Diagnostik nach ICD-10 - Möglichkeiten und Grenzen für die Psychotherapie/Psychosomatik. Psychotherapeut 39:269-275

Stieglitz RD, Freyberger HJ (2013) DSM-5 und ICD-11: Was kommt auf uns zu? Psychother Psychosom Med Psychol 63:207-207

Stieglitz RD, Hiller W (2013) Definition und Erfassung psychischer Störungen. Psychotherapeut 58:237248

\section{Schwerpunktthemen}

Das Herausgebergremium der Zeitschrift Psychotherapeut lädt Autorinnen und Autoren ein, an den geplanten Schwerpunkten mitzuarbeiten und geeignete Manuskripte einzureichen. Diese werden dem üblichen Reviewverfahren unterzogen. Darüber hinaus freuen wir uns über die Zusendung freier (unaufgeforderter) Originalia zu selbstgewählten Themen.

Bitte schicken Sie Ihren Beitrag an die Redaktion:

Regine.Karcher-Reiners@springer.com

Die Schwerpunktplanung ist vorläufig und kann kurzfristig umdisponiert werden.

Heft 3/15: Der psychotherapeutische Prozess

(Deadline 30.12.2014)

Heft 4/15: Psychotherapie der Persönlichkeitsstörungen

(Deadline 30.02.2015)

Heft 5/15: 30 Jahre "Psychotherapeut". Die Zukunft der Psychotherapie (Deadline 30.04.2015)

Heft 6/15: Körperbild (Deadline 30.06.2015)

Heft 1/16: Liebesbeziehungen (Deadline 30.08.2015) 